\title{
Medical training in Belgium: a year in Brussels
}

\author{
W.S.A. Smellie \\ Department of Biochemistry, Glasgow Royal Infirmary, Castle Street, \\ Glasgow G4 OSF, UK.
}

\section{Introduction}

Much of the internal debate on medical training relates to education in posts, progression into speciality subjects and to the workload of junior staff, the latter now accepted to be excessive in many cases. Little general information is available concerning training in other countries, making it difficult to offer constructive criticism, on a comparative basis.

The European Exchange scheme, ${ }^{1}$ run between five London centres and their counterparts in Europe, offers exchange possibilities for up to a year in general medical specialities and allows direct experience of such systems.

In my case my Belgian host institution, Cliniques Universitaires St. Luc, Brussels, has several differences in training from our British system. The hospital forms part of the University of Louvin, and is one of the major Belgian teaching centres.

\section{Medical training}

Medical education at St. Luc is highly structured. The medical degree is seven years long, the last two of which are spent on stagiere rotation through different specialties much as in Britain. During this time medical students have responsibility for preparing definitive admission clerkings and for helping on-ward management, in a role between house physician and student in Britain. They are supervised by the assistant (trainee specialist) answering to a consultant. There remains a major emphasis on teaching in addition to the completion of routine ward work.

Around the time of qualifying a student decides on a career path, a decision which may be difficult to reverse. Those heading for general practice may set up as such immediately following qualification, although vocational training schemes are approaching. Those wishing to enter a speciality are selected by means of an examination with a quota defined for any subject according to the number of training posts available. The examination takes the form of a written paper set

Correspondence: W.S.A. Smellie, M.A., M.R.C.P. and marked by the medical school, to select candidates for postgraduate training in each subject. In general, if unsuccessful in the examination, a candidate would make no further effort to enter training in that subject. Although, as with vocational training schemes for general practice in this country, it is possible to construct an independent training scheme, these were effectively being phased out during my exchange. Selection varies between subjects; for example, success in the examination was around fifty per cent in general medicine in 1987/8.

Specialist training lasts five years and is coordinated by the Professor of Medicine at the teaching centre, with regular review and discussion. An attempt is made to place candidates according to their wishes on one hand and availability on the other. Inevitably this system was criticized as imperfect, although many expressed surprise on hearing of our lack of a coordinated selection system in Britain.

During the five years, training is often begun at peripheral centres with almost all assistants at St. Luc in their third year or above. In the later years trainees are directed towards the chosen speciality, in posts generally of six months duration. Practical techniques in the hospital are mostly grouped into specific posts such as technical cardiology or gastroenterology.

At the end of the period the majority find posts in other hospitals or set up in private or group practice. A number committed to academic medicine stay as resident running wards in conjunction with a consultant, whilst consolidating training or research interests and waiting for a career post to become available. More recently a period of one or two years in research has become expected particularly for academic posts but is still not considered a pre-requisite for nonacademic posts. The role of consultant at St. Luc is similar to that in a British teaching centre with overall responsibility for a number of in-patient beds and for out-patient clinics.

Once selected, and assuming satisfactory progress, progression to career grade is the rule. Ninety per cent of the postgraduates completing training in internal medicine in 1988 found posts immediately whilst some 
of the remaining decided to take time out before applying.

\section{Workload}

Patient throughput is rapid and investigations extensive. As St. Luc is involved mostly in cold investigative medicine, which takes priority over casualty admissions, there is considerable experience for the investigation and management of detailed problems. If the hospital is full, casualty patients are transferred where practical to neighbouring clinics.

The level of debate is extremely high and opportunities to discuss cases with consultant staff excellent, both through formal presentation and particularly informally. There were more formal presentations than in my previous experience, with most units having open weekly meetings in addition to departmental and hospital weekly gatherings. Student teaching commitments of a hospital assistant are similar to those of a registrar in a British teaching hospital. Hours of work are long partly because of time spent in meetings and case conferences. In general medicine however there is a huge reduction in overnight on-call work as compared to the situation in Britain. One assistant manages casualty and one the medical wards with a number available at home for advice for specialist units. Staffing rotas for high dependency areas are organized on a separate basis where on call hours are comparable to a one in three rota to allow continuity of care.

Much of the reduction is related to the effective working of a nursing system. Nurses are trained to insert and manage venous lines, administer drugs, and take blood samples. They are able therefore to manage wards without the continuous presence of junior medical staff. This allows one assistant to manage medical casualty admissions safely with back-up available from ITU teams, and for one in-house assistant to manage many wards without unsafe manning ratios. Arriving, as I did, as an inexperienced senior house officer, the system seemed considerably more efficient than any I had yet experienced, and the working relations on ward generally better than in the United Kingdom, although there are defined demarcation limits between groups of staff.

\section{Conclusions}

The facilities at St. Luc are considered somewhat luxurious by Belgian standards, and do not reflect

\section{References}

1. van Ypersele de Strihou, C. \& Dickinson, C.J. The European Exchange Scheme for junior doctors in internal medicine: an exciting and successful experiment. Lancet 1986, ii: $1446-1448$. those in the country overall. This is, however, a major Belgian teaching establishment. Belgium has more doctors per capita, and a greater health budget than Britain. $^{2}$ These factors may certainly relate to the ability of the system to place staff after the training period, and to increase patient throughput. The funding of the system at St. Luc further encourages increased patient throughput, as hospital income is directly related to the number of patients investigated and treated.

The system in operation at St. Luc has a number of features fundamentally different from the British system. In the first place there is early selection of candidates for a speciality with numbers restricted to availability from the outset. There was effectively no mid-training bottleneck.

Another difference is the use of a co-ordinated training programme supervised by the medical school, and under regular review. There is no need for junior staff to find new posts at six monthly or yearly intervals, as is the current practice for many in Britain.

There was preservation of 'cold' beds allowing a breadth of training and in-patient investigation of non-emergency problems. On my return to a general medical post in London, I found that elective admissions constituted around five per cent of the workload as almost all beds were occupied by casualty admissions.

In St. Luc there are extended facilities for forma and informal training during and outwith working hours, coupled with a need for less on-ward maintenance by junior medical staff. In most British centres junior staff are in constant demand to site, resite and manage venous lines and have limited opportunity to attend postgraduate meetings.

St. Luc also provides an extension of the nursing role to include management of infusions, drugs, and blood sampling, allowing wards to function more autonomously. This is appreciated by both medical and nursing staff.

The different approaches to medical education at St. Luc may not be considered feasible or even desirable in Britain and they are certainly not intended to constitute a shopping list for British medical schools. It is, however, of interest to know that they can operate to good effect in other systems. I found the comparison of medical training an enlightening aspect of the exchange scheme. Such comparisons may in addition become more relevant to our medical schools as some rationalization of training develops between European countries leading up to and after 1992.

2. Maxwell, R.J. Financing health care: lessons from abroad. Br Med J 1988, 296: 1423-1426. 